\title{
Disturbed galaxy clusters are more abundant in an X-ray volume-limited sample
}

\author{
Gayoung Chon and Hans Böhringer
}

\author{
Max-Planck-Institut für extraterrestrische Physik, 85748 Garching, Germany \\ e-mail: gchon@mpe.mpg.de
}

Received 29 August 2017 / Accepted 11 September 2017

\begin{abstract}
X-ray observations of clusters of galaxies have been used to study the large-scale structure of our Universe and to test cosmological models. In such studies it is critical to understand the unique survey selection function correctly. In comparison to the cluster detection by the Sunyaev-Zel'dovich effect (SZE), it has been shown that X-ray observations preferentially detect clusters that have cool cores or are more relaxed as opposed to more disturbed or non-cool-core clusters found in SZE surveys. In this Letter we show that it is not the means of detection, X-rays or SZE, but the sampling strategy, flux-limited or volume-limited surveying, that makes the difference. XMM-Newton observations of the REFLEX clusters in our Volume-Limited Sample (ReVols) show that the fraction of disturbed clusters, determined by the third moment of the power ratios and by centre shifts, is larger by about a factor of two than that of relaxed clusters. In contrast, two flux-limited cluster samples that can be constructed out of ReVols contain more comparable fractions of disturbed and relaxed clusters, which differ by only ten per cent. We use the ratio of the luminosity measured within $r_{500}$ to that measured in the same aperture without the core region as an indicator for a cool core and find that the number of non-coolcore clusters is comparable to or larger than that of the cool-core clusters in ReVols. In addition, we show that the X-ray luminosity distributions of the disturbed and relaxed clusters are distinctly different, and on average, a displacement of $60 \%$ in luminosity is required to match two distributions. Therefore the larger fraction of relaxed and cool-core clusters reported in previous X-ray surveys does not result from the X-ray detection per se, but from the fact that these samples were constructed from flux-limited surveys. Our findings also suggest that the Malmquist bias correction used in cosmological studies with X-ray galaxy clusters could be improved by taking the morphological fractions of the galaxy cluster population and their distinct scaling relations into account.
\end{abstract}

Key words. galaxies: clusters: general - galaxies: clusters: intracluster medium - cosmology: observations $\mathrm{X}$-rays: galaxies: clusters - interplanetary medium

\section{Introduction}

Clusters of galaxies are highly biased peaks of the underlying dark matter distribution, allowing us to study the large-scale structure of our Universe. Because their growth is directly influenced by cosmology, we can also use them to test cosmological models with a cluster survey, which has a well-understood selection function. Among several detection techniques, X-ray observations of clusters reveal the hot intra-cluster plasma that fills the cluster potential that is mostly shaped by dark matter. Hence $\mathrm{X}$-ray observations of clusters provide a powerful method to detect and characterise the cluster population. Equally powerful is a cluster detection through the Sunyaev-Zel'dovich effect (SZE). The same plasma is traced in the cluster potential, but with a different dependence on the gas density. Since SZE is almost insensitive to the redshift dimming, unlike X-ray observations, which are mostly dominated by Bremsstrahlung emission, an SZE survey selection is effectively mass-limited rather than fluxlimited as in a typical X-ray survey. Because the strength of the $\mathrm{X}$-ray signal is proportional to the square of the electron density, an X-ray flux-limited survey has a higher detection efficiency for clusters that are brighter at the centre given the same mass (Pesce et al. 1990; Markevitch 1998). This effect is responsible for a large part of the scatter in the X-ray luminosity-mass relation (Pratt et al. 2009; Chon et al. 2012), which leads to a significant Malmquist bias in a flux-limited sample. This Malmquist bias effect should also lead to an enhancement of the fraction of relaxed and cool-core clusters in flux-limited samples, which can be understood as follows. Most of the clusters in a flux-limited survey are detected near the flux-limit. A scatter of the X-ray luminosity for a given mass will bring more clusters at the more luminous end of the $L_{X}$ distribution into the sample, which is enriched in cool-core clusters, and it will tend to miss clusters at the less luminous end of the distribution, where predominantly disturbed and non-cool cores are located. Because of this effect, studies have shown that flux-limited X-ray samples have a so-called cool-core bias (e.g. Eckert et al. 2011; Rossetti et al. 2017; Andrade-Santos et al. 2017). Therefore it is very important to explore the results that are obtained when this strong Malmquist-type of bias effect can be avoided, for example, in a survey that is volume-limited. Hence we devised a program to study this effect with a volume-limited sample drawn from the REFLEX cluster survey. In this Letter we investigate the morphologies of the clusters in this sample using a combination of centre shifts and the third moment of the power ratios and compare to the properties of the clusters in a flux-limited sample. We also use X-ray luminosity ratios between two different apertures to measure the fraction of cool cores and the X-ray luminosity distributions of the clusters with different morphologies.

In Sect. 2 we describe our study sample and data reduction. The structural analysis and its results are presented in Sect. 3, and discussions on the cool-core fraction are found in Sect. 4 . 
Section 5 investigates X-ray luminosity distributions of clusters with different morphological types. The last section summarises our results and provides a perspective for future work.

\section{Sample selection and data reduction}

We constructed a volume-limited sample (VLS) from the flux-limited REFLEX cluster survey (Chon \& Böhringer 2012; Böhringer et al. 2013) with a redshift limit of $z \leq 0.1$ and a luminosity limit of $5 \times 10^{43} \mathrm{erg} \mathrm{s}^{-1}$ in the $0.1-2.4 \mathrm{keV}$ energy band (rest frame). This sample, which we name ReVols (REFLEXVolume-limited sample), is one of the largest VLSs that can be constructed from REFLEX with a relatively high cluster density. We thinned out this sample statistically by selecting only every third cluster in the third least luminous bin for an affordable $X M M$-Newton follow-up. In addition, we constructed two fluxlimited samples (FLS) within this volume for a comparison between a VLS and an FLS. FLS1 was constructed with a flux limit of $10^{-11} \mathrm{erg} \mathrm{s}^{-1} \mathrm{~cm}^{-2}$ and FLS2 with $1.3 \times 10^{-11} \mathrm{erg} \mathrm{s}^{-1} \mathrm{~cm}^{-2}$. Technically, an FLS should not be constrained in redshift, hence our numerical values in this Letter include additional ten clusters that are above the redshift limit of ReVols. More details on the construction of this sample and the catalogue with the properties of clusters are being prepared (Böhringer et al., in prep.). We obtained relatively deep XMM-Newton observations as well as data from the XMM-Newton archive for a total of 93 clusters. The exposure of individual observations was designed to collect at least 4000 photons inside $r_{500}$ of each cluster for reliable structural measurements. All archival data satisfy this minimum criterium.

We processed the XMM-Newton data as described in Chon et al. (2012). For the clusters whose $r_{500}$ is larger than the field-of-view of XMM-Newton, we used ROSAT PSPC observations to model the cluster emission and estimated the background in the XMM-Newton data. We comment on these clusters in the analysis section.

\section{Structural analysis}

To quantitatively determine the degree of substructure, we employed two common substructure measures: power ratios (Buote \& Tsai 1995), and centre shifts (Poole et al. 2006). They are well tested for X-ray observations and simulations (see e.g. Böhringer et al. 2010; Chon et al. 2012, 2016; Mahdavi et al. 2013; Rasia et al. 2013) and have been shown to provide very useful diagnostics.

\subsection{Power ratio calculation}

The power ratio method first introduced by Buote \& Tsai (1995) was motivated by the assumption that the X-ray surface brightness closely traces the projected two-dimensional mass distribution of a cluster. A multipole decomposition of such a projected mass distribution provides moments that are identified as power ratios after normalisation by the zeroth moment. In practice, the power ratio analysis is applied to the surface brightness distribution.

The moments $P_{m}$ are defined as

$$
\begin{aligned}
& P_{0}=\left[a_{0} \ln \left(R_{\mathrm{ap}}\right)\right]^{2} \\
& P_{m}=\frac{1}{2 m^{2} R_{\mathrm{ap}}^{2 m}}\left(a_{m}^{2}+b_{m}^{2}\right),
\end{aligned}
$$

where $R_{\text {ap }}$ is the aperture radius in units of $r_{500}$. The moments $a_{m}$ and $b_{m}$ are calculated by

$a_{m}(r)=\int_{r \leq R_{\mathrm{ap}}} \mathrm{d} \boldsymbol{x} S(\boldsymbol{x}) r^{m} \cos (m \phi)$

and

$b_{m}(r)=\int_{r \leq R_{\mathrm{ap}}} \mathrm{d} \boldsymbol{x} S(\boldsymbol{x}) r^{m} \sin (m \phi)$,

where $S(\boldsymbol{x})$ is the X-ray surface brightness image, and the integral extends over all pixels inside the aperture radius. Thus, $a_{0}$ in Eq. (1) is the total radiation intensity inside the aperture radius.

Since all $P_{m}$ are proportional to the total intensity of the $\mathrm{X}$-ray image, all moments are normalised by $P_{0}$, resulting in the so-called power ratios, $P_{m} / P_{0}$. For brevity, we refer to $P_{m} / P_{0}$ as $P_{m}$ in the rest of the Letter.

We used $P_{3}$ as one measure of the substructure degree since it is the lowest moment that measures geometric asymmetries above an ellipticity. We calculated the uncertainty of the power ratio measurement and the influence of photon noise with endto-end Monte Carlo simulations in which an additional Poisson noise was imposed on the count images with background. We interpreted the variance of the power ratio results from the simulations as the measurement uncertainty and subtracted the additional noise found in the mean of all simulations compared to the observations from the observational result. Further technical discussions are found in Chon et al. (2012).

\subsection{Centre shifts}

The centre shift measures the stability of the X-ray centre calculated at different radii and is formulated as (Poole et al. 2006)

$w=\left[\frac{1}{N-1} \sum\left(\Delta_{i}-\langle\Delta\rangle\right)^{2}\right]^{1 / 2} \times \frac{1}{r_{500}}$,

where $\Delta_{i}$ is the distance between the mean centroid and the centroid of the $i$ th aperture.

The centroid of each aperture is found by determining the centre of mass of the photon distribution within this aperture. The resulting $w$ is then the standard deviation of the different centre shifts (in units of $r_{500}$ ). We used the mean centroid value of all apertures as the reference centre.

The uncertainties in the $w$ parameter were determined with the same simulations as those of the power ratios, that is, by using Poissonised resampled cluster X-ray images. The standard deviation of the $w$ parameter in the simulation was used as an estimate of the measurement uncertainties. We did not use the noise-bias-subtracted $w$ parameter as in the case of the power ratios since the bias correction is mostly much smaller than the errors and the bias correction does not shift the $w$ parameter to alter the classification of the cluster morphology.

The end-to-end simulations for the power ratios and centre shifts ensures that, for example, the systematics introduced by the photon shot noise is properly taken into account in the parameter uncertainties.

\subsection{Morphological classification}

The measured $w$ and $P_{3}$ values for the ReVols clusters are shown in Fig. 1. Both parameters are generally correlated with some scatter. We note that for the clusters for which $r_{500}$ is not fully 


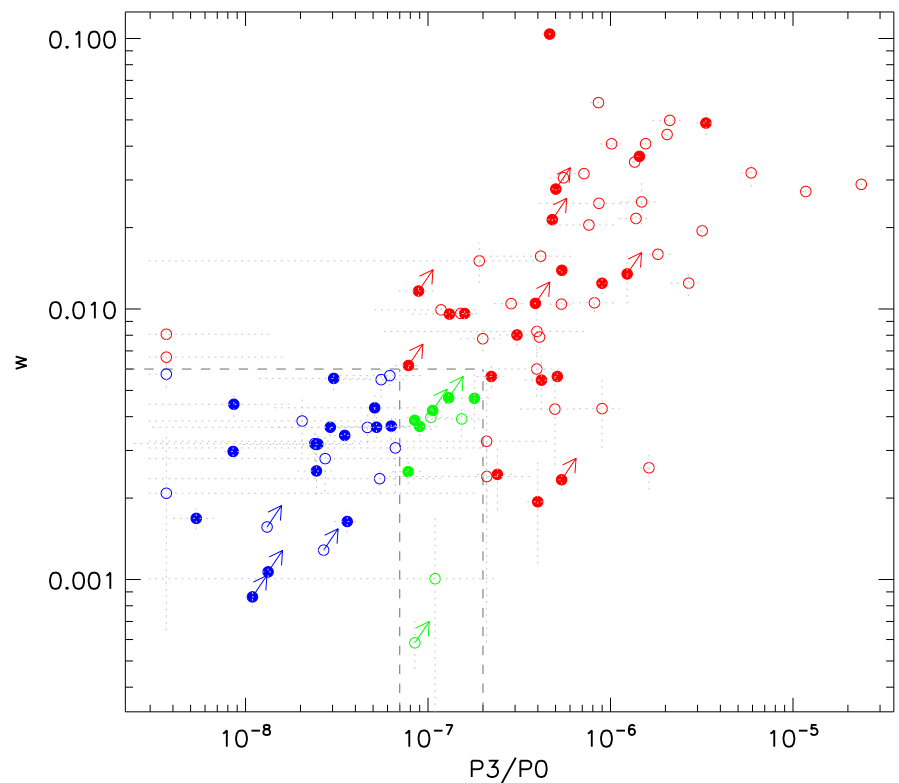

Fig. 1. Structural parameters, $w$ vs. $P_{3}$, for the distributions of the disturbed (red), intermediate (green), and relaxed (blue) clusters. Solid circles are the clusters that also belong to FLS1 above a flux limit of $10^{-11} \mathrm{erg} \mathrm{s}^{-1} \mathrm{~cm}^{-2}$. For the clusters whose $r_{500}$ is not covered by the $X M M-N e w t o n$ observations, the measured values are lower limits, as indicated by upward arrows. The three morphological classifications are based on the three dashed lines.

Table 1. Morphological classification in our VLS and two FLSs.

\begin{tabular}{llll}
\hline \hline Morphology & VLS & FLS1 & FLS2 \\
\hline Disturbed & 56 & 23 & 19 \\
Relaxed & 27 & 21 & 18 \\
\hline Total & 93 & 51 & 42 \\
\hline
\end{tabular}

Notes. FLS1 is constructed with the flux limit of $10^{-11}$ and FLS2 with $1.3 \times 10^{-11} \mathrm{erg} \mathrm{s}^{-1} \mathrm{~cm}^{-2}$. The number of clusters in the intermediate class is the difference between the total and the sum of the disturbed and relaxed clusters.

covered by the XMM-Newton observations, the structural analysis was only calculated out to the minimum available fraction of $r_{500}$ measured from the centre of the aperture. This is to ensure that no artificial asymmetry is introduced when the aperture does not fully cover the cluster. Hence in these cases, the measured $w$ and $P_{3}$ are lower limits of the true values since we normalised both parameters by $r_{500}$ instead of their available fraction, and they are represented by upward arrows.

As in Chon et al. (2012, 2016), we defined three boundaries to classify the clusters into three distinct categories: disturbed $\left(w>0.006\right.$ or $\left.P_{3}>2 \times 10^{-7}\right)$, intermediate $(w<0.006$ and $\left.7 \times 10^{-8}<P_{3}<2 \times 10^{-7}\right)$, and relaxed $\left(w<0.006\right.$ and $P_{3}<$ $\left.7 \times 10^{-8}\right)$. The number of clusters based on this classification is listed in Table 1 under the heading VLS, and the same information is given for two flux-limited samples that are constructed from ReVols, namely FLS1 and FLS2. For comparison, FLS1 clusters are represented by the filled circles in Fig. 1. There are twice more disturbed clusters than relaxed clusters in ReVols, while the two FLSs have more comparable number statistics for both populations. Figure 1 and Table 1 show that the average morphology of the clusters in the volume-limited sample is very different to those found in FLSs.

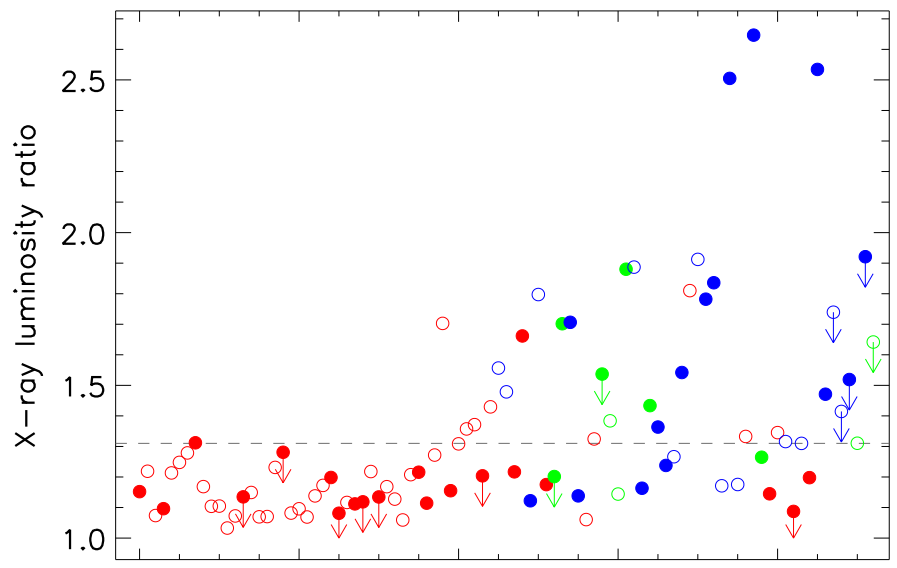

w

Fig. 2. Luminosity ratio of ReVols clusters (all) in comparison to a FLS1 (solid) ordered by $w$ values, which decrease from left to right. Colours follow the morphological classification assigned in Fig. 1. For the clusters whose $r_{500}$ is larger than the XMM-Newton field-of-view the measured ratio is an upper limit, as indicated by downward arrows. The dashed line is drawn at the value of 1.31 , which divides the clusters into $\mathrm{CC}$ and non-CC.

Table 2. Number of clusters in each class.

\begin{tabular}{llll}
\hline \hline & VLS & FLS1 & FLS2 \\
\hline Non-CC & 57 & 27 & 22 \\
Cool core & 36 & 24 & 20 \\
\hline Total & 93 & 51 & 42 \\
\hline
\end{tabular}

Notes. The samples are identical to those described in Table 1.

\section{Cool-core fraction}

The fraction of cool cores (CC) in an X-ray cluster sample was used to study the degree of CC bias and as an indicator for cluster dynamics. Because a CC cluster does not necessarily indicate a dynamically relaxed state, we simply make a comparison of the fraction of CCs in the flux- and volume-limited samples.

The cores of clusters in ReVols, defined by $0.1 \times r_{500}$, are well resolved in the XMM-Newton observations. Hence we calculated the luminosity ratio between the total luminosity in the aperture out to the clusters $r_{500}$ and that measured in the same aperture without the core region (Böhringer et al. 2010). Analogous to the substructure calculations, $r_{500}$ was not fully covered in the observations for some largest clusters. In this case, the surface brightness ratio was calculated again out to the available aperture and was normalised to $r_{500}$. Since the core was always observed, the luminosity ratio becomes an upper limit, and this is indicated by a downward arrow in Fig. 2. We adopted the nominal luminosity ratio of 1.31 as used in Böhringer et al. (2010) to divide the CC population and the non-CCs. Figure 2 and Table 2 show that there are more non-CC clusters in ReVols, while the FLS clusters (filled circles) have more comparable number statistics between the two populations.

\section{Distinct distributions of X-ray luminosity}

Since we expect that $\mathrm{CC}$ clusters are on average more luminous than non-CC clusters for a given cluster mass, we studied the distributions of the X-ray luminosity in more detail for our sample. 


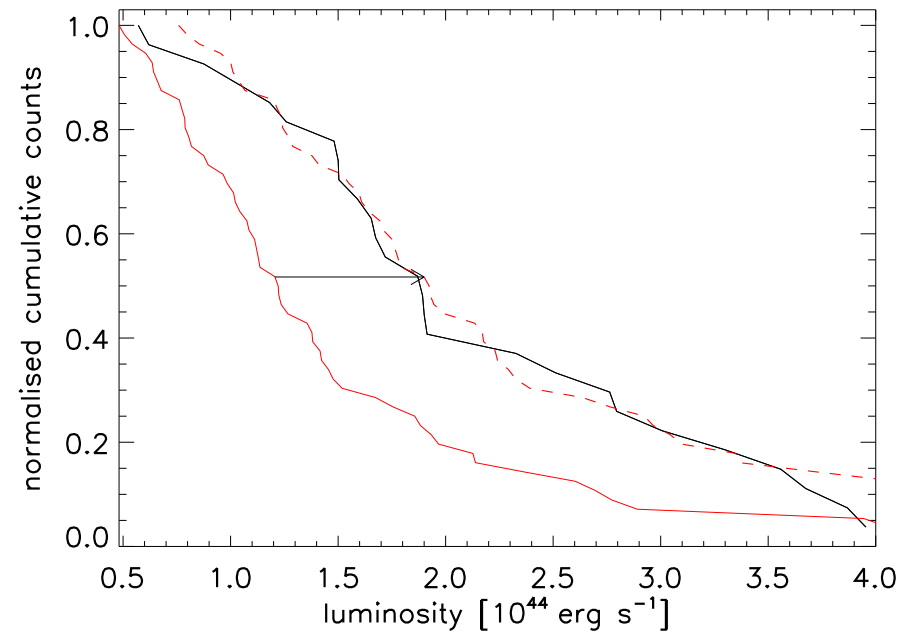

Fig. 3. Normalised cumulative luminosity functions for the disturbed (red) and relaxed (black) clusters. The red dashed line represents the distribution for the disturbed clusters scaled by 1.57 .

Figure 3 shows two normalised cumulative X-ray luminosity distributions for the disturbed clusters as a red line and for the relaxed clusters as a black line. The two populations have distinctly different luminosity distributions, from which we can deduce that there are relatively more luminous clusters in the sample of relaxed clusters than in the disturbed cluster sample. A Kolmogorov-Smirnoff (KS) test shows that it is highly unlikely that both distributions are the same, with a probability of 0.0003 . This difference in the luminosity distribution can have several reasons. When we assume that the mass distribution is not drastically different for the two populations (as found, for example, for the REXCESS sample and in simulations, Böhringer et al. 2010), we could attribute this effect mostly to the luminosity difference for a given mass. In this case, we expect that the two distributions show a similar shape with a constant displacement. Applying a chi-square fit yields a displacement factor of 1.57, as shown by the red dashed line in Fig. 3. To avoid edge effects, we fitted in the luminosity range between 0.7 and $3.7 \times 10^{44} \mathrm{erg} \mathrm{s}^{-1}$. The KS test also finds a best-fit value of 1.59 that maximises the probability, that is, the value that minimises the difference between the two distributions. These results imply that the difference in the luminosity distribution can be explained if relaxed clusters are typically approximately $60 \%$ more luminous than the disturbed clusters.

An approximate factor of 1.6 can in fact be derived from the scaling relations that we presented in Table 2 of Chon et al. (2012). We fitted scaling relations by dividing the clusters according to their morphological classification, finding that there are differences of $40 \%$ and $20 \%$ in the amplitude of the $L_{X}-T$ and in the $M_{\mathrm{X}}-T$ relations, respectively, for a fixed slope. Since the two differences add, this implies that overall we expect a difference of about $60 \%$ in the luminosity between the two populations for a given mass. It is likely that this is not the only effect that contributes to what we observe in ReVols, and in a forthcoming paper we will present scaling relations, which will provide a more complete picture.

\section{Summary and discussions}

We used two measures of substructures, centre shifts and the third moment of the power ratios, to diagnose the degree of substructures in X-ray clusters for our ReVols and two fluxlimited samples derived from REFLEX clusters. As far as we are aware, we present the first numerical evidence that clusters in a volume-limited sample are different from a flux-limited sample in morphology and in the cool-core fraction with relatively large number statistics. We find twice more disturbed than relaxed clusters, and CCs do not dominate ReVols. Thus we do not find that the VLS has a cool-core bias in comparison to cluster populations from SZE surveys. The so-called cool-core bias is therefore found in X-ray cluster samples that are compiled in a flux-limited way.

In the application of X-ray flux-limited cluster samples for cosmological studies, the use of the scaling relation between $\mathrm{X}$-ray luminosity and mass is an important ingredient, and the correction for Malmquist bias related to the scatter in the relation is a prerequisite. In our previous study, we showed that clusters with different morphologies or dynamical states easily influence scaling relations, as demonstrated in Figs. 11 and 12 of Chon et al. (2012). In the standard correction for Malmquist bias, the overall scatter of the $L_{\mathrm{X}}-M$ relation is taken into account regardless of morphological types, and the scatter is typically assumed to have a Gaussian distribution. Our findings suggest a different way of Malmquist-bias correction that may lead to a higher precision in the results because the distribution of the scatter for a mixed morphological population does not appear to be Gaussian. Therefore a procedure where the relation and scatter are determined independently for disturbed and relaxed clusters while taking the morphological fractions also into account may provide a more precise Malmquist-bias correction. As an alternative to this approach, the excision of the cool-core region has been suggested as early as Markevitch (1998). For surveys where the core of clusters cannot be resolved, however, such as the RASS, our suggested refinement of bias correction should provide an improvement. Moreover, in the future, the eROSITA survey does not have the imaging resolution for the clusters at medium and larger distances to precisely excise the core, and the suggested procedure may help to improve the efficiency of the cosmological test.

Acknowledgements. Our research is based on the XMM-Newton facility and data archive operated by ESA. We thank for the support of the XMM-Newton team, especially Norbert Schartel, Ignacio de la Calle, and Maria Santos-Lleo. H.B. and G.C. acknowledge support from the DFG Transregio Program TR33 and the Munich Excellence Cluster "Structure and Evolution of the Universe". G.C. acknowledges support by the Deutsches Zentrum für Luft- und Raumfahrt under grant No. 50 OR 1601 .

\section{References}

Andrade-Santos, F., Jones, C., Forman, W. R., et al. 2017, ApJ, 843, 76 Böhringer, H., Pratt, G. W., Arnaud, M., et al. 2010, A\&A, 514, A32 Böhringer, H., Chon, G., Collins, C. A., et al. 2013, A\&A, 555, A30 Buote, D. A., \& Tsai, J. C. 1995, ApJ, 452, 522

Chon, G., \& Böhringer, H. 2012, A\&A, 538, A35

Chon, G., Böhringer, H., \& Smith, G. P. 2012, A\&A, 548, A59

Chon, G., Puchwein, E., \& Böhringer, H. 2016, A\&A, 592, A46

Eckert, D., Molendi, S., \& Paltani, S. 2011, A\&A, 526, A79

Mahdavi, A., Hoekstra, H., Babul, A., et al. 2013, ApJ, 767, 116

Markevitch, M. 1998, ApJ, 504, 27

Pesce, J. E., Fabian, A. C., Edge, A. C., \& Johnstone, R. M. 1990, MNRAS, 244, 58

Poole, G. B., Fardal, M. A., Babul, A., et al. 2006, MNRAS, 373, 881

Pratt, G. W., Croston, J. H., Arnaud, M., \& Böhringer, H. 2009, A\&A, 498, 361

Rasia, E., Meneghetti, M., \& Ettori, S. 2013, Astron. Rev., 8, 40

Rossetti, M., Gastaldello, F., Eckert, D., et al. 2017, MNRAS, 468, 1917 